\title{
Cálculo del impacto económico para Chile del uso de losartán en diabéticos tipo 2 con nefropatía diabética, basado en resultados del estudio REN AAL
}

\author{
Fernando G onzález $\mathrm{F}^{1,2}$, Verónica Fuentes $\mathrm{C}^{1 \mathrm{a}}$, \\ Catalina Castro $\mathrm{H}^{2}$, Juan Pablo Santelices $\mathrm{L}^{2}$, \\ Eduardo Lorca $\mathrm{H}^{1,2}$. \\ Economic impact of Losartan \\ use in type 2 diabetic patients \\ with nephropathy
}

Background: The study RENAAL (Reduction of Endpoints in NIDDM with the Angiotensin II Antagonist Losartan) demonstrated that Losartan was more effective to reduce the progression of kidney disease in diabetic patients with proteinuria and a reduction in glomerular filtration rate. Aim: To perform a cost benefit analysis of Losartan use from provider and payer points of view. Material and methods: Published data of the RENAAL study was analyzed. The costs of the use or not use of Losartan in patients with diabetic nephropathy were compared in terms of total costs of the disease including medications, hospital admissions for myocardial infarction, cerebrovascular accidents and congestive cardiac failure and the costs of chronic hemodialysis. Results: The reduction in antihypertensive medication use, hospital admissions, and the delay in dialysis requirement from a mean of 65 to 79 months induced by Losartan use, results in net savings of \$7,576,135 per patient, at 3.5 years of intervention. The figure does not change using different sensitivity scenarios. Conclusions: The eventual use of Losartan in type 2 diabetic patients results in important savings (Rev Méd Chile 2009; 137: 634-40).

(Key words: Diabetic nephopathy; Diabetes mellitus, Type 2; Losartan)

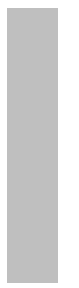

\footnotetext{
Recibido el 21 de noviembre, 2008. Aceptado el 30 de marzo, 2009.

Sin financiamiento externo.

Conflicto de intereses: Fernando González participó como co-investigador en el estudio RENAAL, el que fue diseñado, monitoreado, analizado y financiado por el Laboratorio Merck, Sharp \& Dohme, tal como se describe en referencia 4.

${ }^{1}$ Hospital del Salvador, ${ }^{2}$ Facultad de Medicina, Campus Oriente, Universidad de Chile, Santiago, Chile.

aResidente de Medicina Interna
}

Correspondencia a: Dr. Fernando González Fuenzalida. Departamento de Nefrología, Hospital del Salvador. Av.

Salvador 364, Providencia, Santiago, Chile. Fono-Fax: 269

1205. E mail: fgonzalf@uc.cl 
$E^{1}$ número de pacientes sometidos a hemodiálisis crónica en Chile, a consecuencia de insuficiencia renal terminal, sobrepasan los $12.000^{1}$ y la incidencia de esta condición está aumentando ${ }^{2}$. La principal causa de insuficiencia renal en Chile es la nefropatía diabética, la cual da cuenta de más de $30 \%$ de los casos ${ }^{1}$.

Entre las intervenciones terapéuticas para reducir la progresión de la nefropatía diabética se cuentan el control de la presión arterial y la reducción de la proteinuria, además del control metabólico de la diabetes, la corrección de las dislipidemias asociadas y la suspensión del hábito tabáquico ${ }^{3}$. La interrupción del eje renina angiotensina se ha demostrado particularmente útil, tanto en la nefropatía asociada a diabetes tipo 1 como a la tipo 2, de ahí que los fármacos de elección para estos pacientes son los inhibidores de la enzima de conversión de angiotensina (IECA, por ejemplo: enalapril) y los antagonistas de los receptores de angiotensina II (ARAII, por ejemplo: losartán) ${ }^{3}$.

El principal estudio que avala el uso de los ARAII en nefropatía diabética es el RENAAL (Reduction of Endpoints in NIDDM with the Angiotensin II Antagonist Losartan), publicado en el año $2001^{4}$ y que incluyó pacientes chilenos del Hospital del Salvador. En él, se demostró que en diabéticos tipo 2 con macroproteinuria y reducción de la velocidad de filtración glomerular, losartán $\left(\right.$ Cozaar $^{\circledR}$, Merck, Sharp \& Dohme) era más efectivo que la terapia antihipertensiva convencional para prevenir la progresión de la insuficiencia renal, el ingreso a diálisis o el fallecimiento prematuro, además que reducía la incidencia de problemas o eventos cardiovasculares mayores, como por ejemplo, hospitalizaciones por insuficiencia cardiaca congestiva ${ }^{4}$.

El uso de losartán en Chile, en pacientes como los que participaron en el estudio RENAAL, es poco común, posiblemente porque el costo del fármaco es mayor que el de otras drogas de uso clínico habitual. Aunque se han realizado estudios de costos de la incorporación de losartán en pacientes con nefropatía diabética en varios países $^{5-12}$, ellos no necesariamente se aplican a la realidad chilena y tampoco consideran la evaluación del impacto de la reducción de las hospitalizaciones sobre el costo total.

La valorización económica de las hospitalizaciones de pacientes complejos es difícil, debido a la realización de múltiples procedimientos diagnósticos y terapéuticos, al uso de un amplio arsenal farmacológico y problemas logísticos inherentes al costeo unitario de las prestaciones realizadas en este tipo de enfermos. En 1983, el Medicare de Estados Unidos de Norteamérica comenzó a emplear el sistema de valorización asociado a diagnósticos o GRD (Grupos Relacionados a Diagnósticos) que consiste en agrupar pacientes portadores de iguales diagnósticos, según la Clasificación Internacional de Diagnósticos, y que utilizan cantidades de recursos similares cuando se hospitalizan, simplificando de esta forma las valorizaciones ${ }^{13}$.

El Hospital del Salvador utiliza el sistema de GRD para valorizar las hospitalizaciones, de tal forma que es posible conocer cuánto cuestan al sistema de salud las internaciones según los diagnósticos de alta de los pacientes.

Nuestro objetivo es realizar un análisis formal de costos del uso de losartán en pacientes portadores de nefropatía diabética utilizando los datos publicados del estudio RENAAL, dado que sus resultados podrían ser representativos de nuestra población. Un segundo objetivo es realizar este estudio desde la óptica del prestador y pagador ambulatorio (consultorios de atención primaria), del prestador y pagador de las hospitalizaciones (Hospital del Salvador) y del pagador de la diálisis (Fondo Nacional de Salud o Fonasa), puesto que son entidades diferentes, con asignaciones de recursos independientes, perspectivas de uso de ellos y horizontes de intervención distintos entre sí.

\section{MATERIAL Y MÉTODO}

Datos para el análisis. De la publicación del New England Journal of Medicine del estudio RENAAL ${ }^{4}$ se extrajeron los datos numéricos relevantes para este análisis: probabilidades de uso de familias de fármacos antihipertensivos (losartán o placebo), frecuencias observadas de hospitalizaciones por puntos finales cardiovasculares secundarios relevantes (insuficiencia cardiaca congestiva, infarto agudo al miocardio y accidente vascular encefálico) y pendientes del recíproco de la creatininemia plasmática. Esta última, para calcular el tiempo requerido desde el reclutamiento de los pacientes participantes en el estudio y una velocidad de 
filtración glomerular de $12 \mathrm{ml} / \mathrm{min}$, según fórmula de Crockoft-Gault, que definimos como aquélla que requiere de terapia de sustitución de la función renal.

De los registros de internaciones del Hospital del Salvador, se seleccionó aleatoriamente al menos 25 pacientes que cumplían con todos los criterios de inclusión y exclusión del estudio RENAAL y que habían estado internados, ya sea por insuficiencia cardiaca congestiva, infarto agudo al miocardio o accidente vascular encefálico. De las hospitalizaciones de estos enfermos (25, 33 y 29 casos, respectivamente), se extrajo el cálculo de GRD realizado por el Hospital para así estimar sus costos.

El costo de la diálisis es el correspondiente a la modalidad institucional de Fonasa y el de los fármacos antihipertensivos, es el precio de compra de ellos y que se obtuvo de la farmacia del hospital.

Todos los valores monetarios son en moneda del año 2006.

Análisis de resultados. Con la información numérica obtenida de las fuentes antes descritas, se construyó la función de costo en la que se incorporan las probabilidades de uso de cada familia de fármacos, incluyendo losartán o placebo, con sus costes y período de uso, las probabilidades de observación de cada evento cardiovascular con sus costos asociados y el número de meses de requerimiento de terapia de sustitución de la función renal en cada grupo de intervención.

Se comparó las funciones de costo de utilizar losartán o placebo, lo que es equivalente a comparar el costo de cada una de esas dos estrategias de intervención.

El análisis anterior puede describirse gráficamente como un árbol de decisiones de Markov ${ }^{14}$.

$$
\begin{aligned}
& \mathrm{F}(\text { costo })_{\mathrm{Los}}=\mathrm{P}_{\mathrm{Los}}{ }^{*} \mathrm{C}_{\mathrm{Los}}{ }^{*} \mathrm{M}_{\mathrm{Los}}+\Sigma_{\mathrm{i}=1}{ }^{\mathrm{n}} \mathrm{P}_{\mathrm{AHi}}{ }^{*} \mathrm{C}_{\mathrm{AHi}}{ }^{*} \mathrm{M}_{\mathrm{AHi}}+ \\
& \Sigma_{\mathrm{j}=1}{ }^{n} \mathrm{P}_{\mathrm{HCVj}}{ }^{*} \mathrm{C}_{\mathrm{HCVj}}{ }^{*} \mathrm{~F}_{\mathrm{HCVj}}+\mathrm{P}_{\mathrm{HD}}{ }^{*} \mathrm{C}_{\mathrm{HD}}{ }^{*} \mathrm{M}_{\mathrm{HD}} \\
& \mathrm{F}(\text { costo })_{\mathrm{Pla}}=\mathrm{P}_{\mathrm{Pla}}{ }^{*} \mathrm{C}_{\mathrm{Pla}}{ }^{*} \mathrm{M}_{\mathrm{Pla}}+\Sigma_{\mathrm{i}=1}{ }^{\mathrm{n}} \mathrm{P}_{\mathrm{AHi}}{ }^{*} \mathrm{C}_{\mathrm{AHi}} * \mathrm{M}_{\mathrm{AHi}}+ \\
& \Sigma_{\mathrm{j}=1}{ }^{n} \mathrm{P}_{\mathrm{HCVj}}{ }^{*} \mathrm{C}_{\mathrm{HCVj}}{ }^{*} \mathrm{~F}_{\mathrm{HCVj}}+\mathrm{C}_{\mathrm{HD}}{ }^{*} \mathrm{M}_{\mathrm{HD}}
\end{aligned}
$$

Donde:

$\mathrm{F}(\text { costo })_{\mathrm{Los} / \mathrm{Pla}}=$ Función de costo de losartán o placebo.
$\mathrm{P}_{\mathrm{Los}^{\prime}} \mathrm{P}_{\mathrm{Pla}^{\prime}} \mathrm{P}_{\mathrm{AHi}^{\prime}} \mathrm{P}_{\mathrm{HCVj}}=$ Probabilidades de: uso de losartán, placebo, otro antihipertensivo u hospitalización por complicación cardiovascular j.

$\mathrm{C}_{\mathrm{Ios}^{\prime}} \mathrm{C}_{\mathrm{Pla}}=\quad$ Costos de losartán o placebo.

$\mathrm{M}_{\text {Los, }} \mathrm{M}_{\text {Pla }}=\quad$ Meses de uso de losartán o placebo.

$\Sigma_{\mathrm{i}=1}{ }^{\mathrm{n}} \mathrm{C}_{\mathrm{AHi}}{ }^{*} \mathrm{M}_{\mathrm{AHi}}=$ Sumatoria del producto de los costos de cada familia de antihipertensivos y sus meses de uso.

$\Sigma_{\mathrm{j}=1}{ }^{\mathrm{n}} \mathrm{C}_{\mathrm{HCVj}}{ }^{*} \mathrm{~F}_{\mathrm{HCVj}}=$ Sumatoria del producto del costo GRD de hospitalizaciones por causa cardiovascular y sus respectivas frecuencias.

$\mathrm{C}_{\mathrm{HD}}=\quad$ Costo de la hemodiálisis.

$\mathrm{M}_{\mathrm{HD}}=\quad$ Meses de hemodiálisis.

Análisis de sensibilidad. Para el análisis preliminar de resultados se utilizó las probabilidades publicadas en el estudio RENAAL de uso de losartán o placebo, las probabilidades de uso de familias de fármacos antihipertensivos y las frecuencias de ocurrencias de complicaciones cardiovasculares. Lo mismo que las pendientes de los recíprocos de las creatininemias y los promedios de los costos GRD de las hospitalizaciones por complicaciones cardiovasculares.

El análisis de sensibilidad se efectuó en varias etapas: 1. Variando los precios de compra de losartán y de los fármacos antihipertensivos. 2. Usando los extremos de los intervalos de confianza a $95 \%$ de los grupos losartán o placebo para cada probabilidad o frecuencia, de tal forma de maximizar y minimizar las diferencias entre ambos grupos. 3. Realizando programación lineal mediante el programa computacional Solver (Excel 2003 de Microsoft Corporation ${ }^{\circledR}$ ) para resolver la 0 las condiciones en las cuales la estrategia de utilizar placebo es menos cara que utilizar losartán.

\section{Resultados}

En el estudio RENAAL ${ }^{4}$ participaron 1.513 personas (751 en el grupo losartán). Ambos grupos tuvieron características basales similares al inicio del estudio en cuanto a sexo, edad, raza, índice de masa corporal, presión arterial, comorbilidad cardiovascular o relacionada a complicaciones de la 
diabetes mellitus y variables de laboratorio, como hemoglobina A1c, niveles de colesterol total y triglicéridos, creatininemia y proteinuria cuantificada por cuociente proteinuria/creatininuria.

Las probabilidades de uso de fármacos antihipertensivos y las frecuencias de hospitalizaciones por complicaciones cardiovasculares en dicho estudio se muestran en las Tablas 1 y 2. Las pendientes de las curvas de los recíprocos de las creatininemias fueron $0,056 \mathrm{dL} / \mathrm{mg} /$ año en el grupo losartán y 0,069 dL/mg/año en el grupo placebo $(p=0,01)$.

El número de meses estimado para alcanzar una velocidad de filtración glomerular, según fórmula de Crockoft-Gault, de $12 \mathrm{ml} / \mathrm{min} \mathrm{y}$, por tanto, llegar al umbral de requerimiento de terapia de sustitución de la función renal fue 79,3 meses en el grupo losartán y 64,6 meses en el grupo placebo.

Los costos GRD de las complicaciones cardiovasculares fueron: $1,71 \pm 0,52$ para la insuficiencia cardiaca, 1,72 $\pm 0,51$ para el infarto al miocardio $\mathrm{y}$ $1,55 \pm 0,39$ para el accidente vascular encefálico. Cada GRD tenía un valor de $\$ 400.000$.

En la Tabla 3 puede observarse las funciones de costo de las dos estrategias terapéuticas. Los hechos más destacables de ella son que el costo total de fármacos antihipertensivos es mayor en la estrategia losartán, sin embargo, como ella se asocia a menor gasto por hospitalizaciones por eventos cardiovasculares, el costo total sin diálisis de ambas estrategias es prácticamente igual. El costo asociado a terapia de sustitución de la función renal es \$7,6 millones en total.

El análisis de sensibilidad mostró que: 1. La modificación de los costos de cualquiera de las familias de fármacos antihipertensivos no modifica significativamente los resultados mostrados en la Tabla 3. En este sentido, el costo diario de losartán que equilibra las dos estrategias de intervención es \$3.195. 2. Usando los extremos de los intervalos de confianza a 95\% de los grupos losartán o placebo para cada probabilidad o frecuencia, de tal forma de maximizar y minimizar las diferencias entre ambos grupos, tampoco modifica los resultados mostrados. En estos casos, el costo de losartán de equilibrio de las dos estrategias terapéuticas es \$ 3.217 y \$ 3.076, respectivamente. 3. El análisis por programación lineal determinó que en ninguna circunstancia se modifica el hallazgo central que la estrategia losartán es la más económica.

Tabla 1. Frecuencias de uso de fármacos antihipertensivos convencionales en el estudio REN AAL ${ }^{4}$

\begin{tabular}{|lcc|}
\hline & Losartán & Placebo \\
\hline Calcio antagonistas total & 0,78 & 0,81 \\
No dihidropiridínicos & 0,17 & 0,17 \\
Dihidropiridínicos & 0,61 & 0,64 \\
Diuréticos & 0,84 & 0,84 \\
Alfabloqueadores & 0,40 & 0,46 \\
Betabloqueadores & 0,34 & 0,37 \\
Agentes centrales & 0,18 & 0,22 \\
\hline
\end{tabular}

Tabla 2. Freauencias de ocurrencia de hospitalizaciones por complicaciones cardiovasculares en el estudio REN AAL ${ }^{4}$

\begin{tabular}{|lcr|}
\hline & Losartán & Placebo \\
\hline Insuficiencia cardiaca & 0,12 & 0,17 \\
Infarto miocardio & 0,07 & 0,09 \\
Accidente vascular encefálico & 0,06 & 0,07 \\
\hline
\end{tabular}


Tabla 3. Funciones de costo de las intervenciones terapéuticas expresadas como costo por paciente durante todo el período de seguimiento

\begin{tabular}{|lrr|}
\hline & Losartán & Placebo \\
\hline Costos antihipertensivos & $\mathbf{\$}$ & $\mathbf{\$}$ \\
Costo losartán & 96.097 & 108,560 \\
Costo fármacos total (A) & 72.828 & 0 \\
Hospitalizaciones por complicaciones cardiovasculares (GRD) & 168.925 & 108.560 \\
Costo GRD & 0,625 & 0,790 \\
Costo hospitalizaciones total (B) & 400.000 & 400,000 \\
Meses de diálisis & 250.000 & 316.000 \\
Costo mensual diálisis & 0 & 14,7 \\
Costo diálisis total (C) & 515.000 & 515.000 \\
Costo total sin diálisis (A+B) & 0 & 7.570 .500 \\
Costo total con diálisis (A+B+C) & 418.925 & 424.560 \\
\hline
\end{tabular}

Los determinantes de costo del costo total de la estrategia losartán son los fármacos antihipertensivos (46,5\%) y el losartán (35\%), mientras que en la estrategia conservadora es la diálisis (89,4\%).

\section{DisCUSIÓN}

Al igual que en los análisis fármaco-económicos realizados en otros países y con distintas metodologías a la nuestra ${ }^{5-12}$, la estrategia terapéutica más conveniente en pacientes que sufren de nefropatía secundaria a diabetes mellitus tipo 2 en fase macroproteinúrica y con deterioro de la velocidad de filtración glomerular es el bloqueo del eje renina angiotensina mediante el uso de losartán. El beneficio conseguido deriva de la reducción de la progresión de la enfermedad renal crónica y de una menor frecuencia de complicaciones cardiovasculares que determinan hospitalizaciones, como son la insuficiencia cardiaca congestiva, el infarto al miocardio y los accidentes vasculares encefálicos.

El costo diario de losartán, bajo la óptica del resultado poblacional global de la intervención en nefropatía diabética, parece irrelevante si se lo compara con el alto costo de la terapia de sustitución de la función renal. Gráficamente, si se ahorra un solo mes de diálisis, se consigue un presupuesto aproximado de $\$ 515.000$ para invertir en otras intervenciones terapéuticas, como son, por ejemplo, un mejor control metabólico de la diabetes, terapia hipolipemiante o programas que ayuden en detener el hábito tabáquico de los fumadores.

Los análisis de sensibilidad realizados son categóricos en demostrar que la estrategia de intervención con losartán es la más conveniente desde el punto de vista económico. En el mismo sentido, el análisis de los determinantes del costo total de las dos estrategias de intervención informa que una vez utilizado losartán, la única preocupación del clínico debe ser, desde el punto de vista económico, el intentar un control adecuado de la presión arterial, en cambio, si la estrategia decidida es no prescribir losartán, se deberá estar conciente que el costo será alto y que no existen intervenciones que permitan un ahorro real, como no sea el restringir el acceso a diálisis a quienes lo requieran, lo cual, evidentemente crea conflictos éticos serios.

El losartán es una droga que bloquea el eje renina angiotensina, al igual que los inhibidores de la enzima de conversión de angiotensina (IECA) u otros bloqueadores de los receptores de angiotensina II. Cabe preguntarse si el uso de, por ejemplo, enalapril o valsartan, por nombrar sólo algunos otros medicamentos, puede conseguir 
resultados análogos a los encontrados en el estudio RENAAL y en nuestro análisis. Desde el punto de vista ortodoxo $\mathrm{y}$, fundamentalmente, desde el económico, la respuesta parece ser que, primero, se necesita de estudios de intervención farmacológicos con puntos finales de importancia clínica y, segundo, que sus respectivos análisis económicos sean superiores 0 al menos equivalentes a lo observado por nosotros. Es posible que el bloqueo del eje renina angiotensina con IECAs sea una alternativa al uso de losartán ${ }^{15}$, dado que ambos son antiproteinúricos y la reducción de la proteinuria es un objetivo prioritario en el tratamiento de estos enfermos ${ }^{16}$.

Fortalezas de nuestro estudio son el haber dispuesto de información publicada que incluye a 26 pacientes locales y el contar con metodología validada para la estimación de los costos, particularmente el GRD de las principales causas de hospitalización de estos enfermos y que son el estándar de nuestro centro hospitalario. Sin embargo, es claro que un análisis de costos será limitado toda vez que no incluya todos los costos indirectos, por ejemplo, el costo económico derivado de dejar de trabajar durante las hospitalizaciones y la merma en la capacidad productiva de quienes sufren de insuficiencia renal terminal y necesitan dializarse. No obstante lo anterior, intuitivamente, pareciera que estos aspectos más bien amplifican la utilidad del losartán.

Cabe preguntarse el por qué es, a priori, difícil que estudios como éste tengan el impacto que debieran en las políticas de salud. La razón más

\section{REFERENCIAS}

1. Pobiete H. XXVII Cuenta de Hemodiálisis Crónica en Chile al 31 de agosto de 2007. Sociedad Chilena de Nefrología. http://www.asodi.cl/c_h_c_2007_ok.pdf. [Accedido el 28 de diciembre de 2008].

2. GonZÁlez F. La implementación del Plan de Acceso Universal y Garantías Explícitas (plan AUGE) ha deteriorado la calidad del tratamiento de pacientes con insuficiencia renal terminal. Rev Méd Chile 2006; 134: $1288-94$

3. GonzÁlez F. Nefropatía Diabética. En Contreras C, editor. Santiago, Chile: Editorial Mediterráneo SA; 2004; 259-92.

4. Brenner B, Cooper M, De Zeeuw D, Grunfeid JP, Keane W, Parving HH et al, For the RENAAL Study InvestigaTORS. Effects of losartan on renal and cardiovascular probable es que las transferencias de recursos económicos entre los distintos pagadores de los servicios de la salud no son para nada fáciles. A modo de ejemplificar lo anterior, tenemos que la incorporación de losartán a la atención primaria 0 secundaria debe ser financiada con fondos de la atención ambulatoria y los ahorros conseguidos en hospitalizaciones y en diálisis serán a favor de los presupuestos de los hospitales y del Fondo Nacional de Salud (Fonasa). Aun cuando el dinero total provenga del erario nacional, las distribuciones de los recursos económicos no necesariamente consideran los ahorros cruzados que puedan aparecer con la implementación de nuevas intervenciones terapéuticas.

En Chile, se está analizando la posibilidad de incorporar la prevención de la enfermedad renal crónica (ERC) al llamado Plan AUGE o GES, y de integrarlo al Programa de Salud Cardiovascular del MINSAL, lo que significa que: 1) seguramente, aumentará el número de pacientes que requieran una intervención terapéutica destinada a retardar la progresión de la enfermedad hacia la insuficiencia renal terminal, tal como ocurrió en el año 2002 cuando comenzó el plan piloto $\mathrm{AUGE}^{17}$, y 2) es posible que pueda mejorar el impacto sobre la morbilidad y mortalidad cardiovascular en etapas más tempranas de la ERC, como se sugiere en este estudio. Es fundamental el estudiar la incorporación de medicamentos que sean eficaces en esta tarea y no negarse, a priori, a ellos por consideraciones de costo que, eventualmente, pueden, a la postre, resultar erradas.

outcomes in patients with type 2 diabetes and nephropathy. N Engl J Med 2001; 345: 861-9.

5. Seng WK, Hwang SJ, Han DC, Teong CC, Chan J, BURKE TA ET AL. Losartan reduces the costs of diabetic end-stage renal disease: an Asian perspective. Nephrology (Carlton) 2005; 10: 520-4.

6. Arredondo A, Burke TA, Carides GW, Lemus E, Querol $\mathrm{J}$. The impact of losartan on the lifetime incidence of ESRD and costs in Mexico. Rev Invest Clin 2005; 57: 399-405.

7. Alexander CM, Lyle Pa, Keane WF, Carides GW, Zhang Z, Shahinfar S. Losartan and the United States costs of end-stage renal disease by baseline albuminuria in patients with type 2 diabetes and nephropathy. Kidney Int Suppl 2004; 92: S115-7.

8. Szucs TD, Sandoz MS, Keusch GW. The cost-effectiveness of losartan in type 2 diabetics with nephropa- 
thy in Switzerland-an analysis of the RENAAL study. Swiss Med Wkly 2004; 7; 134: 440-7.

9. Burgess ED, Carides GW, Gerth WC, Marentette MA, Снавот I. Losartan reduces the costs associated with nephropathy and end-stage renal disease from type 2 diabetes: Economic evaluation of the RENAAL study from a Canadian perspective. Can J Cardiol 2004; 20: 613-8.

10. Souchet $T$, Durand Zaleski $I$, Hannedouche $T$, $\backslash 0$ "Click to search for citations by this author." RoDIER M, \o "Click to search for citations by this author." Gaugris S, 10 "Click to search for citations by this author." PASSA P ET AL. An economic evaluation of Losartan therapy in type 2 diabetic patients with nephropathy: an analysis of the RENAAL study adapted to France. Diabetes Metab 2003; 29: 29-35.

11. Herman W, Shahinfar S, Carides G, Dasbach E, Gerth W, Alexander C et al. Losartan Reduces the Costs Associated With Diabetic End-Stage Renal Disease. The RENAAL study economic evaluation. Diabetes Care 2003; 26: 683-7.

12. Gerth WC, Remuzzi G, Viberti G, , Martinez-Castelao A, Shahinfar S ET AL. Losartan reduces the burden and cost of ESRD: Public health implications from the RENAAL study for the European Union. Kidney Int 2002; 62 Suppl 82: 68-72.

13. http://www.ahrq.gov/data/hcup/94drga.htm [Accedido el 29 de diciembre de 2006].

14. Bala M, Mauskopf J. Optimal Assignment of Treatments to Health States Using a Markov Decision Model: An Introduction to Basic Concepts. Pharmacoeconomics 2006; 24: 345-54.

15. Barnett A, Bain S, Bouter P, Karlberg B, Madsbad S, JERVELL J ET AL. Angiotensin-Receptor Blockade versus Converting-Enzyme Inhibition in Type 2 Diabetes and Nephropathy. N Eng J Med 2004; 351: 1952-61.

16. Eijkeikamp W, Zhang Z, Remuzzi G, Parking H-H, Cooper M, Keane W ET aL Albuminuria Is a Target for Renoprotective Therapy Independent from Blood Pressure in Patients with Type 2 Diabetic Nephropathy: Post Hoc Analysis from the Reduction of Endpoints in NIDDM with the Angiotensin II Antagonist Losartan (RENAAL) Trial. J Am Soc Nephrol 2007; 18: 1540-6.

17. GonZÁLEZ F. Implementación del plan AUGE en pacientes con IRC. Rev Méd Chile 2003; 131: 545-51. 